\title{
Discoid lupus erythematosus co-existent with vitiligo patches on the scalp: A case report
}

\section{Hassanandani Trashita, Behera Debasmita, Panda Maitreyee, Debata Ipsita}

\author{
Department of Dermatology, Venerealogy and Leprosy, IMS \& SUM Hospital, Bhubaneswar, Odisha, India
}

Corresponding author: Dr. Hassanandani Trashita, E-mail: htrashita@gmail.com

\begin{abstract}
Discoid lupus erythematosus, a type of chronic cutaneous lupus erythematosus presents as scaly plaques mostly over sun exposed parts of the body. Vitiligo is characterized by depigmented patches. Co-existence of the two is a rare phenomenon. There have been few reports of DLE coexisting with vitiligo over acral parts but to the best of our knowledge none of them were present over the scalp. Here we present a case of coexistence of DLE and vitiligo lesions over the scalp of an adult male and the challenge in therapy of the two conditions occurring simultaneously over the scalp.
\end{abstract}

Key words: Discoid lupus erythematosus; Vitiligo; Scalp

\section{INTRODUCTION}

Discoidlupus erythematosus (DLE) is the most common form of chronic cutaneous lupus erythematosus. It is characterized by scaly plaques which heal with atrophy and scarring mostly over the sun exposed areas. Vitiligo results from progressive loss of melanocytes leading to depigmented milky white patches. It is an autoimmune condition and has been associated with various other autoimmune diseases such as alopecia areata, thyroid disease and rarely systemic lupus erythematosus. However, DLE lesions developing with vitiligo patches is a rarer finding specially over the scalp and the presence of one disorder may affect the treatment of the other [1]. Here we present a case of coexistence of DLE and vitiligo lesions over the scalp of an adult male.

\section{CASE REPORT}

A 41 year old male presented with asymptomatic depigmented areas over the scalp since 1 year which have been increasing in size and number. The lesions on the parietal scalp region had developed redness and crusting over the last 4 months.
On examination, there were multiple depigmented patches with leukotrichia over the scalp. The lesion on the parietal region was erythematous, scaly and indurated with some atrophy in the center (Fig. 1). The patient didn't give any history of application over the area. Family history was negative. There was no associated autoimmune disorder.

Histopathology of the plaque over parietal region showed follicular plugging, perivascular and peri appendageal lymphocytic infiltrate and basal cell degeneration which was consistent with Discoid lupus erythematosus (Fig. 2). Histopathology from depigmented lesion on the temporal scalp showed decreased number of melanocytes suggestive of vitiligo.

Laboratory tests revealed positive ANA. Thyroid function test was done which was negative.

The patient was started on oral prednisolone $(0.5 \mathrm{mg} /$ $\mathrm{kg}$ ) which is a safe and effective modality in the treatment of both DLE and vitiligo where it stops the scarring process of DLE and the progression of vitiligo. The patient was advised strict photoprotection and although vitiligo would have improved with

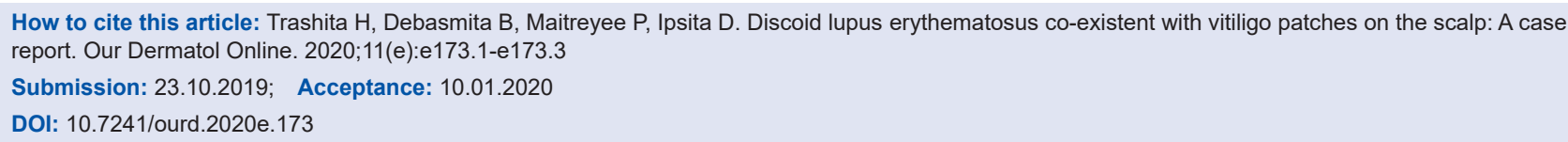




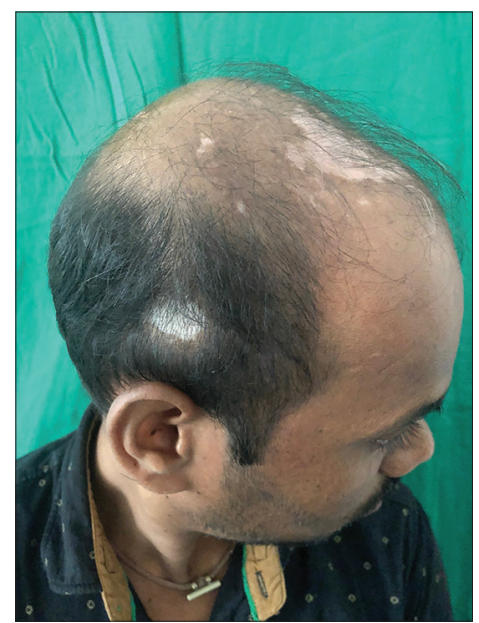

Figure 1: Depigmented patch over temporal scalp (vitiligo), erythematous atrophic patch over parietal scalp (discoid lupus erythematosus).

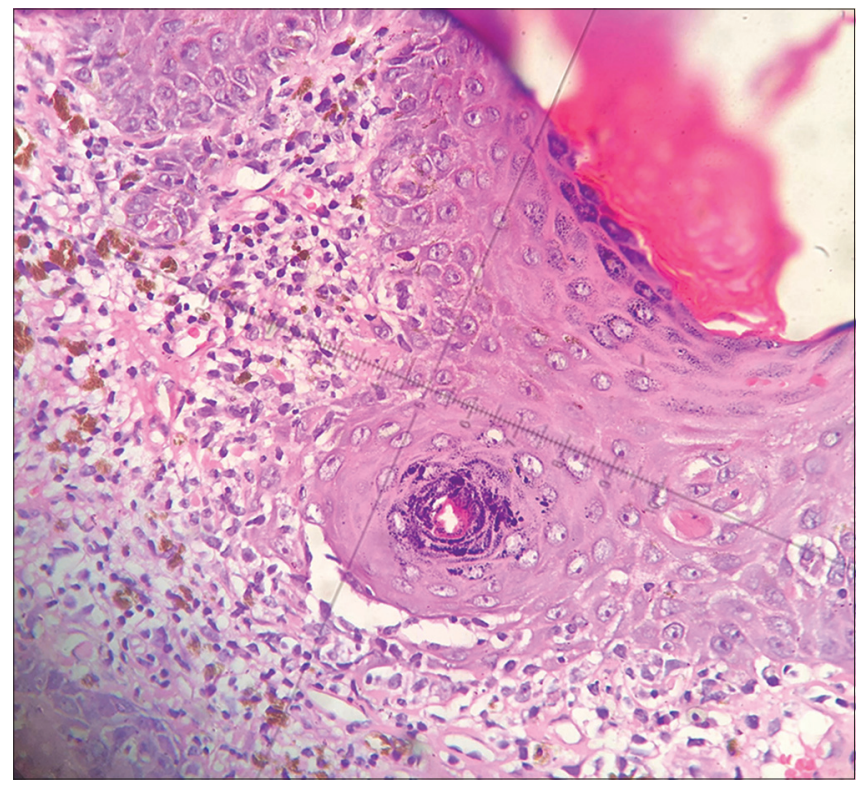

Figure 2: Histopathology showing follicular plugging, perivascular and peri appendageal lymphocytic infiltrate and basal cell degeneration consistent with discoid lupus erythematosus.

phototherapy, no such modality was used as it had a potential risk of aggravating the DLE lesion specially over the photo exposed parts like the scalp. After 3 months of therapy with tapering dose of steroids both the lesions were fairly controlled. The patient was advised to apply topical tacrolimus $0.1 \%$ for maintenance.

\section{DISCUSSION}

Vitiligo has been associated with a spectrum of autoimmune conditions such as alopecia areata, thyroid disease, pernicious anaemia, Addison's disease and rarely systemic lupus erythematosus [2]. Ying Jin et al identified several chromosomal regions that appear to contribute to this epidemiological association including chromosome 17pl3 [3].

In a report by Forestier et al., DLE occurred within the vitiliginous skin on both exposed and non-exposed surfaces where they postulated that localizing role of vitiligo in these exceptional cases could represent a more complex disorder of the dermo-epidermal junction. This disorder could affect more than the melanocytic system and subsequently favour the occurrence of the LE [4]. A similar occurrence was reported quite rarely [5]. Sharma et al reported the coexistence of lip tip vitiligo with DLE which was treated with hydroxychloroquine and topical steroids [1]. To the best of our knowledge there have been no reported cases of isolated scalp involvement. Oral steroids have a promising role in such situations where the treatment of two dermatoses is diametrically different. In such cases it is also imperative to follow up the patient for the increased risk of development of carcinoma.

\section{CONCLUSION}

Coexistence of vitiligo and DLE over the scalp is a rare finding and can pose a therapeutic challenge. Oral steroid is an effective modality in such a case.

\section{Consent}

The examination of the patient was conducted according to the Declaration of Helsinki principles.

The authors certify that they have obtained all appropriate patient consent forms. In the form the patient(s) has/have given his/her/ their consent for his/her/their images and other clinical information to be reported in the journal. The patients understand that their names and initials will not be published and due efforts will be made to conceal their identity, but anonymity cannot be guaranteed.

\section{REFERENCES}

1. Sharma S, Sarkar R, Garg VK, Bansal S. Coexistence of liptip vitiligo and disseminated discoid lupus erythematosus with hypothyroidism: Need for careful therapeutic approach. Indian Dermatol Online J. 2013;4:112-4.

2. Rahimi BA, Farooqi K, Fazli N. Clinical patterns and associated comorbidities of vitiligo in Kandahar, Afghanistan. A case-control study. Our Dermatol Online. 2020;11:6-12.

3. Jin Y, Mailloux CM, Gowan K, Riccardi SL, LaBerge G, Bennett DC, et al. NALP1 in vitiligo-associated multiple autoimmune disease. N Engl J Med. 2007;356:1216-25.

4. Sharma S, Sarkar R, Garg VK, Bansal S. Coexistence of lip- 
www.odermatol.com

tip vitiligo and disseminated discoid lupus erythematosus with hypothyroidism: Need for careful therapeutic approach. Indian Dermatol Online J. 2013;4:112-4.

5. Johnson H, Bossenbroek NM, Rosenman K, Meehan SA, Robles M, Pomeranz MK. Chronic cutaneous lupus erythematosus in vitiligo. Dermatol Online J. 2008 14:10.
Copyright by Hassanandani Trashita, et al. This is an open access article distributed under the terms of the Creative Commons Attribution License, which permits unrestricted use, distribution, and reproduction in any medium, provided the original author and source are credited.

Source of Support: Nil, Conflict of Interest: None declared. 